\title{
Ueber die Entwicklung einer Function von beliebig vielen Variablen nach Laplaceschen Functionen höherer Ordnung.
}

\author{
(Von Herrn F. G. Mehler zu Danzig.)
}

Die beiden Sätze, auf welche man bei der Entwicklung einer Function von zwei Variablen nach Kugelfunctionen die Bestimmung des allgemeinen Gliedes zu gründen pflegt, sind von Herrn Cayley *) auf die von ihm definirten allgemeineren, von beliebig vielen Variablen abhängigen Laplaceschen Functionen übertragen worden. Auf ganz anderem Wege sind diese Sätze später von Herrn Heine in der Arbeit „Die speciellen Laméschen Functionen erster Art von beliebiger 0rdnung(**) abgeleitet, worin man ausserdem eine Reihe von interessanten Eigenschaften der speciellen Functionen findet, welche in der allgemeinen Theorie die Stelle der Kugelfunction $\boldsymbol{P}_{n}(\cos \gamma)$ und ihrer $\mathrm{Zu}-$ geordneten vertreten. Schon vor dem Erscheinen der soeben genannten Arbeit war ich durch Uebertragung der Eigenschaften des Potentials einer Flächenbelegung auf ein vielfaches Integral zu einer Verallgemeinerung der Laplaceschen Reihen gelangt. In dem zu Ostern 1864 ausgegebenen Programme der Realschule zu St. Johann in Danzig habe ich die Form der Reihen für beliebig viele Variablen aufgestellt und den Fall dreier Variablen einer speciellen Discussion unterworfen. Ich sprach dort zugleich meine Absicht aus, in einer späteren Bearbeitung auf die Erörterung der Convergenz der allgemeinen Reihenentwicklungen einzugehen, sowie auch auf die Betrachtung gewisser neuer Reihen, welche aus jenen dadurch hervorgehen, dass man die Zahl der Variablen unendlich gross werden, die zu entwickelnde Function aber nur von einer beschränkten Anzahl derselben in passender Weise abhängen lässt. Die letzteren Reihen, bei welchen die Variablen nicht auf ein bestimmtes Intervall beschränkt sind, sondern alle möglichen reellen Werthe annehmen können, erwiesen sich mir später als ein besonderer Fall der von Herrn Hermite ***) schon am Anfange des Jahres 1864 veröffentlichten Ent-

*) Sur les fonctions de Laplace. Liouvilles Journal Bd. 13.

**) Bd. $62, \mathrm{~S}_{\mathrm{i}} 110-141$ dieses Journals.

***) Sur un nouveau développement en série des fonctions. Comptes Rendus, Bd. 58, S. $93-100$ und $266-273$. 
162 Mehler, Reihenentwicklungen nach Laplaceschen Functionen höherer Ordnung.

wicklungen, indem sie sich aus denselben durch Specialisirung der darin auftretenden quadratischen Form ergeben. Da mir der Nachweis der Verwandtschaft der Reihen des Herrn Hermite mit den verallgemeinerten Laplaceschen Reihen und die Untersuchung der Convergenz der letzteren nicht ohne Wichtigkeit zu sein scheint, so glaube ich, dass die nachfolgenden Mittheilungen auch neben den genannten Arbeiten noch Manches bieten werden, was der Beachtung werth ist. Verschiedene, ohne den gewünschten Erfolg gebliebene Versuche, den auftretenden Convergenzbedingungen allgemein eine einfachere Gestalt zu geben, haben mich von einer früheren Veröffentlichung zurückgehalten.

\section{\$. 1.}

Definition der Laplaceschen Functionen $\boldsymbol{n}^{\text {ter }}$ Ordnung.

Die partielle Differentialgleichung

$$
\text { (1.) } \frac{\partial^{2} v}{\partial x_{1}^{2}}+\frac{\partial^{2} v}{\partial x_{2}^{2}}+\cdots+\frac{\partial^{2} v}{\partial x_{n+1}^{2}}=0
$$

hat, wie bekannt, den reciproken Werth des Ausdruckes

$$
\boldsymbol{R}=\left[\left(x_{1}-a_{1}\right)^{2}+\left(x_{2}-a_{2}\right)^{2}+\cdots+\left(x_{n+1}-a_{n+1}\right)^{2}\right]^{\frac{1}{3(n-1)}}
$$

zum particulären Integral. Es genügt ihr also, wenn unter $K^{\prime}$ eine beliebige endliche Function der $a$ verstanden wird, auch das $(n+1)$ fache Integral

$$
v=\int \frac{K^{\prime} d a_{1} d a_{2} \ldots d a_{n+1}}{R}=\int \frac{K^{\prime} d t^{\prime}}{R},
$$

vorausgesetzt, dass $\boldsymbol{R}$ für keinen innerhalb der Integrationsgrenzen gelegenen Werth der $a$ verschwindet. Führt man nun statt der $x$ die Variablen $\varrho, \varphi_{1}$, $\varphi_{2}, \ldots \varphi_{*}$ ein vermittelst der Gleichungen

$$
\begin{aligned}
& x_{1}=\rho \cos \varphi_{1}, \\
& x_{2}=\rho \sin \varphi_{1} \cos \varphi_{2}, \\
& \cdot \cdot \cdot \cdot \cdot \cdot \cdot \cdot \\
& x_{n}=\rho \sin \varphi_{1} \sin \varphi_{2} \ldots \sin \varphi_{n-1} \cos \varphi_{n}, \\
& x_{n+1}=\rho \sin \varphi_{1} \sin \varphi_{2} \ldots \sin \varphi_{n-1} \sin \varphi_{n},
\end{aligned}
$$

so geht (1.) über in:

$$
\frac{1}{\rho^{n-2}} \frac{\partial}{\partial \varrho}\left(\rho^{n} \frac{\partial v}{\partial \rho}\right)+\sum_{s=1}^{s=n} \frac{1}{q_{s} \sin ^{n-s} \varphi_{s}} \frac{\partial}{\partial \varphi_{s}}\left(\sin ^{n-s} \varphi_{s} \frac{\partial v}{\partial \varphi_{s}}\right)=0
$$

worin $q_{1}=1$, und für $s>1$ :

$$
q_{s}=\left(\sin \varphi_{1} \sin \varphi_{2} \ldots \sin \varphi_{s-1}\right)^{2}
$$


Mehler, Reihenentwicklungen nach Laplaceschen Functionen höherer Ordnung. 163 und wenn man der obigen Substitution entsprechend

und ausserdem

$$
\begin{aligned}
& a_{1}=l \cos \lambda_{1}, \\
& a_{2}=l \sin \lambda_{1} \cos \lambda_{2}, \quad \text { etc. }
\end{aligned}
$$

$$
a_{1} x_{1}+a_{2} x_{2}+\cdots+a_{n+1} x_{n+1}=l \varrho \cdot \cos \omega
$$

setzt, so wird

$$
\begin{aligned}
R & =\left(l^{2}-2 l \varrho \cos \omega+\varrho^{2}\right)^{\frac{1}{2}(n-1)}, \\
d t^{\prime} & =l^{n} \sin ^{n-1} \lambda_{1} \ldots \sin ^{1} \lambda_{n-1} d l d \lambda_{1} \ldots d \lambda_{n-1} d \lambda_{n} .
\end{aligned}
$$

Die Function $v$ soll jetzt so specialisirt werden, dass sie für $n=2$ mit dem Potential einer auf einer Kugel vom Halbmesser 1 vertheilten Massenschicht zusammenfällt. Zu dem Zwecke verwandeln wir $K^{\prime}$ in $k^{\prime}: \varepsilon$, verstehen unter $k^{\prime}$ eine von $l$ unabhängige Function $\operatorname{der} \lambda$, unter $\varepsilon$ eine unendlich kleine Grösse, integriren nach $l$ von 1 bis $1+\varepsilon$ und nehmen 0 und $\pi$ als Integrationsgrenzen von $\lambda_{1}, \ldots \lambda_{n-1}$, dagegen 0 und $2 \pi$ als die von $\lambda_{n}$. Indem wir noch zur Abkürzung

$$
\begin{aligned}
r & =\left(1-2 \varrho \cos \omega+\varrho^{2}\right)^{\frac{1}{2}(n-1)} \\
d \sigma^{\prime} & =\sin ^{n-1} \lambda_{1} \ldots \sin ^{1} \lambda_{n-1} d \lambda_{1} \ldots d \lambda_{n-1} d \lambda_{n}
\end{aligned}
$$

setzen, erhalten wir

$$
\text { (3.) } \quad v=\int \frac{k^{\prime} d \sigma^{\prime}}{r} \text {. }
$$

Bezeichnet nun $\boldsymbol{R}_{m}(\cos \omega)$ den Coefficienten von $\varphi^{m}$ in der Entwicklung von $r^{-1}$ nach aufsteigenden, oder, was dasselbe ist, den von $\varrho^{-m-n+1}$ in der Entwicklung von $r^{-1}$ nach absteigenden Potenzen von $\varrho$, und setzt man

$$
\text { (4.) } \quad \boldsymbol{X}_{m}=\int \dot{k}^{\prime} \boldsymbol{R}_{m}(\cos \omega) d \sigma^{\prime} \text {, }
$$

dann gilt für $v$, je nachdem $\varrho<1$ oder $>1$, die erste oder die zweite der beiden folgenden Reihenentwicklungen:

$$
\begin{aligned}
& \left(3^{\prime} .\right) \quad v=\sum_{m=1}^{m=\infty} \varrho^{m} X_{m}, \\
& \left(3^{\prime \prime} .\right) \quad v=\sum_{m=1}^{m=\infty} \varphi^{-m-n+1} X_{m},
\end{aligned}
$$

und durch Einführung der einen oder der anderen in (2.) ergiebt sich für $X_{m}$ die (schon von Herrn Ieine aufgestellte) partielle Differentialgleichung

$$
m(m+n-1) X_{m}+\sum_{s=1}^{s=n} \frac{1}{q_{s} \sin ^{n-s} \varphi_{s}} \frac{\partial}{\partial \varphi_{s}}\left(\sin ^{n-s} \varphi_{s} \frac{\partial X_{m}}{\partial \varphi_{s}}\right)=0 .
$$


164 Mehler, Reihenentwicklungen nach Laplaceschen Functionen höherer Ordnung.

Eine jede Lösung $Z_{m}$ dieser Differentialgleichung, welche, wie es mit $\boldsymbol{X}_{m}$ der Fall ist, eine ganze rationale Function ( $m^{\text {ten }}$ Grades) der Grössen $\cos \varphi_{1}$, $\sin \varphi_{1} \cos \varphi_{2}$ etc. ist, möge eine Laplacesche Function $n^{\text {ter }}$ Ordnung heissen: unsere Aufgabe besteht dann darin, eine Function von $n$ Variablen $\varphi$, welche für alle Werthe von $\varphi_{1}, \ldots \varphi_{n-1}$ zwischen 0 und $\pi$ und für alle Werthe von $\varphi_{n}$ zwischen 0 und $2 \pi$ gegeben ist, in eine Reihe $Z_{0}+Z_{1}+Z_{2}+\cdots$ von Laplaceschen Functionen $n^{\text {ter }}$ Ordnung zu entwickeln.

\section{\$. 2.}

Ableitung der Reihenentwicklungen.

Für $n=2$ kann die Form der Reihe bekanntlich sehr leicht durch Anwendung des Satzes erbalten werden, dass die zur Kugelfläche normale Attractionscomponente beim Durchgange durch die Fläche eine plötzliche Aenderung um eine Grösse erfährt, die der Dichtigkeit der Massenschicht an der Durchgangsstelle proportional ist. Dem analog suchen wir in dem allgemeinen Falle den Grenzwerth zu ermitteln, welchen die Differenz

$$
\left(\frac{\partial v}{\partial \varrho}\right)_{(\varrho=1+\varepsilon)}-\left(\frac{\partial v}{\partial \varrho}\right)_{(\varrho=1-\varepsilon)}=D
$$

für ein unendlich kleines positives $\varepsilon$ annimmt. Diese Differenz stellt sich vermöge (3.) als der Unterschied zweier vielfachen Integrale dar, deren entsprechende Elemente sich unendlich wenig von einander unterscheiden, mit Ausnahme derjenigen, für welche $r$ der Null sehr nahe liegt. Da dies aber nur für kleine Werthe von $\omega$ stattfindet, so ist es zweckmässig, es so einzurichten, dass $\omega$ eine der Integrationsvariablen wird. Indem wir deshalb von den Variablen $\lambda$ für den Augenblick wieder zu den $a$ zurückgehen, ersetzen wir diese durch $n+1$ neue Variablen $b, b_{1}, \ldots b_{n}$ durch die folgende orthogonale Substitution:

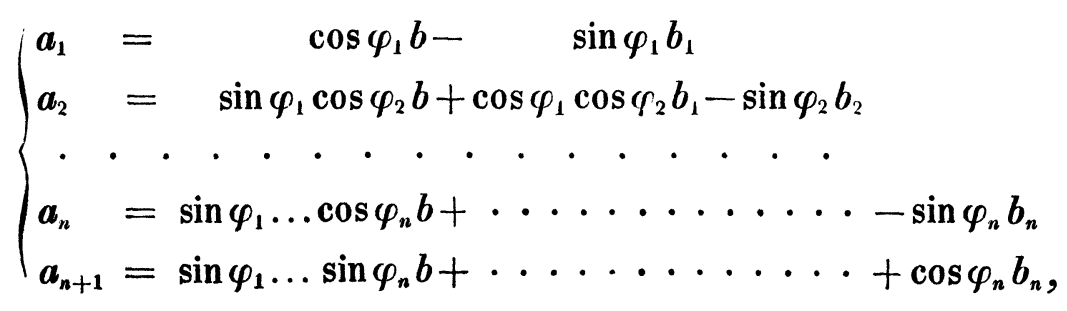

worin die Coefficienten von $b_{i}$ aus denen von $b$ durch partielle Differentiation nach $\varphi_{i}$, unter Fortlassung des gemeinsamen Factors $\sin \varphi_{1} \ldots \sin \varphi_{i-1}$, hervor- 
Mehler, Reihenentwicklungen nach Laplaceschen Functionen höherer Ordnung. 165 gehen. Aus diesen Gleichungen folgt:

$$
b=a_{1} \cos \varphi_{1}+a_{2} \sin \varphi_{1} \cos \varphi_{2}+\cdots=l \cos \omega,
$$

und setzt man ausserdem

$$
b_{1}=l \sin \omega \cos \omega_{1}, \ldots, \quad b_{n}=l \sin \omega \ldots \sin \omega_{n-1},
$$

so wird (3.) transformirt in:

$$
v=\int_{0}^{\pi} \ldots \int_{0}^{2 \pi} F(\varrho) \sin ^{n-2} \omega_{1} \ldots \sin ^{1} \omega_{n-2} d \omega_{1} \ldots d \omega_{n-1},
$$

wenn

$$
F(\rho)=\int_{0}^{\pi} \frac{k^{\prime} \sin ^{n-1} \omega d \omega}{\left(1-2 \varrho \cos \omega+\varrho^{2}\right)^{\frac{1}{2}(n-1)}},
$$

so dass es sich jetzt nur um die Werthbestimmung der Differenz $F^{\prime}(1+\varepsilon)-F^{\prime}(1-\varepsilon)$ handelt. Differentiirt man nun $F(\varrho)$ nach $\varrho$, setzt dann $\varrho=1+\varepsilon$, und zugleich

$$
\cos \omega=1-\frac{1}{2} z^{2}, \quad \frac{1-n}{2}\left(1-\frac{1}{4} z^{2}\right)^{\frac{1}{2} n-1} \cdot k^{\prime}=h^{\prime},
$$

so erhält man:

$$
F^{\prime}(1+\varepsilon)=\int_{0}^{2} \frac{h^{\prime} d z}{\left(1+\frac{\varepsilon^{2}}{z^{2}}+\varepsilon\right)^{\frac{1}{2}(n+1)}}+\int_{0}^{2} \frac{2 \varepsilon h^{\prime} d z}{z^{2}\left(1+\frac{\varepsilon^{2}}{z^{2}}+\varepsilon\right)^{\frac{1}{2}(n+1)}} .
$$

Das erste dieser Integrale ändert sich, wie leicht zu sehen, unendlich wenig, wenn $\varepsilon$ in $-\varepsilon$ verwandelt wird. Es kommt also nur das zweite in Betracht, welches wir in zwei Theilintegrale mit den Grenzen 0 und $\delta, \delta$ und 2 zerlegen, während wir $\delta$ mit $\varepsilon$ zugleich gegen Null hin abnehmen lassen, jedoch so, dass der Quotient $\varepsilon: \delta$ stets unendlich klein bleibt. Das zweite Theilintegral ist, wenn $H$ einen gewissen endlichen Werth bezeichnet, gleich

$$
H \int_{\delta}^{2} \frac{2 \varepsilon d z}{z^{2}}=H\left(\frac{2 \varepsilon}{\delta}-\varepsilon\right)
$$

verschwindet also zugleich mit $\varepsilon$, und das erste ist, wenn $\left(h^{\prime}\right)$ den Werth des $h^{\prime}$ für einen gewissen zwischen 0 und $\delta$ gelegenen Werth $z_{1}$ des $z$ bedeutet, gleich

$$
\left(h^{\prime}\right)\left(1+\frac{\varepsilon}{1+\frac{\varepsilon^{2}}{z_{1}^{2}}}\right)^{-\frac{1}{2}(n+1)} \cdot \int_{0}^{o \delta} \frac{2 \varepsilon d z}{z^{2}\left(1+\frac{\varepsilon^{2}}{z^{2}}\right)^{\frac{1}{2}(n+1)}} .
$$

Aber der Factor von $\left(h^{\prime}\right)$ vor dem Integralzeichen ist unendlich wenig von 1 verschieden, und das Integral geht durch die Substitution $z=\varepsilon t^{-\frac{1}{2}}$ über in:

$$
\int_{\left(\frac{\varepsilon}{\delta}\right)^{2}}^{\infty} \frac{t^{1-1} d t}{(1+t)^{\frac{1}{2}(n+1)}}=\int_{0}^{\infty} \frac{t^{\frac{1}{2}-1} d t}{(1+t)^{\frac{1}{(1(n+1)}}}=\frac{\sqrt{ } \pi \Gamma\left(\frac{1}{2} n\right)}{\Gamma_{\frac{1}{2}(n+1)}(n+1)}
$$


Das Doppelte dieses Werthes mit $\left(h^{\prime}\right)$ oder $\frac{1}{2}(1-n)\left(k^{\prime}\right)$ multiplicirt ist gleich der Differenz $F^{\prime}(1+\varepsilon)-F^{\prime}(1-\varepsilon)$, und daher ist:

$$
D=-\frac{2 \sqrt{ } \pi \Gamma\left(\frac{1}{2} n\right)}{\Gamma \frac{1}{2}(n-1)} \int_{0}^{\cdot \pi} \int_{0}^{2 \pi}\left(k^{\prime}\right) \sin ^{n-2} \omega_{1} \ldots \sin ^{1} \omega_{n-2} d \omega_{1} \ldots d \omega_{n-1} .
$$

Es bedeutete $\left(k^{\prime}\right)$ den Werth des $k^{\prime}$ für ein unendlich kleines $\omega$. Aber für $\omega=0$ folgt aus (6.), dass $\cos \lambda_{1}=\cos \varphi_{1}, \sin \lambda_{1} \cos \lambda_{2}=\sin \varphi_{1} \cos \varphi_{2}$ u. s. w., und hieraus gehen unter der Voraussetzung, dass die $\varphi$ keinen ihrer Grenzwerthe 0 und $\pi$ (resp. 0 und $2 \pi$ ) besitzen, für die $\lambda$ die bestimmten Auflösungen $\lambda_{1}=\varphi_{1}, \lambda_{2}=\varphi_{2}$ u. s. w. hervor, von denen sich die für ein unendlich kleines $\omega$ geltenden nur unendlich wenig unterscheiden. Wenn also die Function $k^{\prime}=f\left(\lambda_{1}, \ldots \lambda_{n}\right)$ für das besondere Werthsystem $\lambda_{1}=\varphi_{1}, \lambda_{2}=\varphi_{2}$ etc. nicht vieldeutig ist, so nähert sich $\left(k^{\prime}\right)$ der von $\omega_{1}, \omega_{2}$ etc. unabhängigen Grenze $k=f\left(\varphi_{1}, \ldots \varphi_{n}\right)$, und die Gleichung (7.) kann dann durch die folgende ersetzt werden:

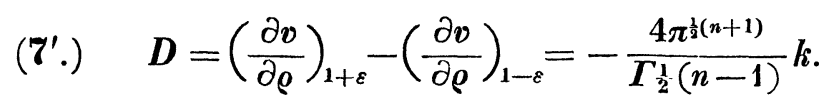

In Betreff der Grenzwerthe der Variablen wird die Bemerkung genügen, dass $D$ für $\varphi_{i}=0$ und $\varphi_{i}=\pi$ von $\varphi_{i+1}, \varphi_{i+2}$ etc. unabhängig, und dass für $\varphi_{n}=0$ und $\varphi_{n}=2 \pi$ an Stelle von $k$ das arithmetische Mittel der diesen beiden Werthen entsprechenden Functionalwerthe zu setzen ist.

Indem man $\left(7^{\prime}\right.$.) mit $\left(3^{\prime \prime}\right.$.) und $\left(3^{\prime}\right.$.) verbindet, darauf $\varrho=1$ und für $X_{m}$ den in (4.) befindlichen Werth setzt, erhält man:

$$
\text { (I.) } \quad k=\frac{\Gamma_{\frac{1}{2}}(n-1)}{4 \pi^{\frac{1}{3}(n+1)}} \sum_{m=0}^{m=\infty}(2 m+n-1) / k^{\prime} R_{m}(\cos \omega) d \sigma^{\prime},
$$

aber die Gültigkeit dieser Entwicklung bleibt zweifelhaft, weil ihre Ableitung auf der Annahme beruht, dass die angewandten nach Potenzen von $\rho$ fortschreitenden Reihen, welche für $\varrho=1+\varepsilon$, resp. $\varrho=1-\varepsilon$, allerdings sicher convergent sind, ihre Convergenz auch noch für $\varrho=1$ beibehalten.

Die in dem allgemeinen Gliede auftretende specielle Laplacesche Function $\boldsymbol{R}_{m}(\cos \omega)$ hat, je nachdem $n$ eine ungerade oder gerade Zahl ist, einen wesentlich verschiedenen Charakter; es ist nämlich, wenn man $z$ statt $\cos \omega$ schreibt,

für $n=2 \mu+1, \mu>0$ :

$$
\boldsymbol{R}_{m}(z)=\frac{1}{(m+\mu) 2^{\mu-1} \Gamma(\mu)} \frac{d^{\mu} \cos (m+\mu) \omega}{d z^{\mu}},
$$


Mehler, Reihenentwicklungen nach Laplaceschen Functionen höherer Ordnung. 167 und für $n=2 u+2, \mu \geqq 0$ :

$$
\boldsymbol{R}_{m}(\boldsymbol{z})=\frac{\sqrt{ } \boldsymbol{\pi}}{2^{\mu} \boldsymbol{\Gamma}\left(\mu+\frac{1}{2}\right)} \frac{d^{\mu} P_{m+\mu}(z)}{d z^{\mu}},
$$

in Betreff welcher Ausdrücke ich auf die oben angeführte Arbeit des Herrn Heine verweise. Die Reihe in (I.) führt also auf die beiden folgenden Reihen :

$$
\begin{aligned}
\left(\mathbf{I}^{a} .\right) \quad S= & \frac{2}{(2 \pi)^{\mu+1}} \sum_{m=0}^{m=\infty} \int f\left(\lambda_{1}, \ldots \lambda_{2 \mu+1}\right) \frac{d^{\mu} \cos (m+\mu) \omega}{d z^{\mu}} d \tau, \\
d \tau= & \sin ^{2 \mu} \lambda_{1} \ldots \sin ^{1} \lambda_{2 \mu} d \lambda_{1} \ldots d \lambda_{2 \mu+1}, \\
z= & \cos \omega=\cos \varphi_{1} \cos \lambda_{1}+\sin \varphi_{1} \sin \lambda_{1} \cos \varphi_{2} \cos \lambda_{2}+\ldots \\
& \quad+\sin \varphi_{1} \sin \lambda_{1} \ldots \sin \varphi_{2 \mu} \sin \lambda_{2 \mu} \cos \left(\varphi_{2 \mu+1}-\lambda_{2 \mu+1}\right) . \\
\left(\mathbf{I}^{b} .\right) \quad S= & \frac{1}{(2 \pi)^{\mu+1}} \sum_{m=0}^{m=\infty}\left(m+\mu+\frac{1}{2}\right) \int f\left(\lambda_{1}, \ldots \lambda_{2 \mu+2}\right) \frac{d^{\mu} P_{m+\mu}(z)}{d z^{\mu}} d \tau .
\end{aligned}
$$

Die Ausdrücke für $d \tau$ und $z$ in der letzten Gleichung gehen aus den voranstehenden durch Verwandlung von $2 \mu$ in $2 \mu+1$ hervor.

\section{\$. 3.}

Ueber die Convergenz der Reihenentwicklungen.

Die wichtigen Arbeiten Dirichlets über die trigonometrischen und die nach Kugelfunctionen fortschreitenden Reihen (im $4^{\text {ten }}$ und $17^{\text {ten }}$ Bande dieses Journals) bieten die hauptsächlichsten Hülfsmittel dar, um auch die erhaltenen allgemeineren Reihen zu untersuchen. Bezeichnet man durch $S_{m}$ die Summe der $m-\mu+1$ ersten Glieder der Reihe $\left(I^{a}.\right)$, in welcher die Function $f$ als endlich vorausgesetzt werde, und bemerkt man, dass

$$
\cos (\mu \omega)+\cos (\mu+1) \omega+\cdots+\cos (m \omega)=\frac{1}{2} \frac{\sin \left(m+\frac{1}{2}\right) \omega}{\sin \frac{1}{2} \omega}-G,
$$

wo $G$ eine ganze Function von $z$ vom $\mu-1^{\text {ten }}$ Grade, so findet man:

$$
S_{m}=\frac{1}{(2 \pi)^{\mu+1}} \int f\left(\lambda_{1}, \ldots \lambda_{2 \mu+1}\right) \frac{d^{\mu}}{d z^{\mu}}\left(\frac{\sin \left(m+\frac{1}{2}\right) \omega}{\sin \frac{1}{2} \omega}\right) d \tau,
$$

und wenn man dieses Integral durch die Substitution (6.) transformirt und

$$
\Psi(\omega)=\int_{0}^{\pi} \ldots \int_{0}^{2 \pi} f\left(\lambda_{1}, \ldots \lambda_{2 \mu+1}\right) \sin ^{2 \mu-1} \omega_{1} \ldots \sin ^{1} \omega_{2 \mu-1} d \omega_{1} \ldots d \omega_{2 \mu}
$$

setzt, so wird :

$$
\text { (A. }) \quad S_{m}=\frac{1}{(2 \pi)^{\mu+1}} \int_{0}^{n} \Psi(\omega) \sin ^{2 \mu} \omega \frac{d^{\mu}}{d z^{\mu}}\left(\frac{\sin \left(m+\frac{1}{2}\right) \omega}{\sin \frac{1}{2} \omega}\right) d \omega .
$$


168 Mehler, Reihenentwicklungen nach Laplaceschen Functionen höherer Ordnung.

Wenn nun die Function $\Psi(\omega) \sin ^{2 \mu-1} \omega$ und ihre nach $z=\cos \omega$ genommenen $\mu-1$ ersten Differentialquotienten innerhalb der Integrationsgrenzen continuirlich bleiben und an den Grenzen selbst verschwinden, so ist es nicht allein erlaubt, $\mu$ mal hinter einander theilweise zu integriren, sondern es verschwinden auch die vom Integralzeichen freien Glieder, so dass man, indem man zur Abkürzung

$$
X(\omega)=\frac{(-1)^{\mu-1}}{2(2 \pi)^{\mu}} \frac{d}{d \omega}\left[\frac{d^{\mu-1} \Psi(\omega) \sin ^{2 \mu-1} \omega}{d z^{\mu-1}}\right]
$$

setzt, für $S_{m}$ den Ausdruck erhält:

$$
\text { (B.) } \quad S_{m}=\frac{1}{\pi} \int_{0}^{\pi} \frac{\sin \left(m+\frac{1}{2}\right) \omega}{\sin \frac{1}{2} \omega} X(\omega) d \omega .
$$

Für ein unendlich grosses $m$ nähert sich derselbe, wie Dirichlet gezeigt hat, sicher dann dem Werthe $X(+0)$, wenn dieser Werth endlich ist und $X(\omega)$ in dem Intervalle von 0 bis $\pi$ weder unendlich viele Stetigkeitsunterbrechungen erleidet noch unendlich viele Maxima und Minima besitzt*). Betrachtet man nun $\Psi(\omega)$ als den Quotienten von $\Psi(\omega) \sin ^{2 \mu-1} \omega$ und $\sin ^{2 \mu-1} \omega$, so ist, weil diese Grössen nebst ihren $\mu-1$ ersten nach $z$ genommenen Differentialquotienten für $\omega=0$ verschwinden, $\Psi(+0)$ gleich $\operatorname{dem}$ Quotienten der $\mu^{\text {ten }}$ Differentialquotienten beider für $\omega=0$, wodurch man leicht findet, dass

$$
\Psi(+0)=\frac{X(+0)}{C}, \text { wenn } \quad C=\frac{\Gamma\left(\mu+\frac{1}{2}\right)}{2 \pi^{\mu+\frac{1}{2}}} .
$$

Es wird also $S_{\infty}=C \Psi(+0)$, und diese Grösse ist aus den schon bei Gelegenheit der Gleichung (7.) des vorigen Paragraphen angeführten Gründen gleich $f\left(\varphi_{1}, \varphi_{2}, \ldots \varphi_{2 \mu+1}\right)$, sofern dieser Werth nicht vieldeutig ist und keine der Variablen einen ihrer Grenzwerthe besitzt.

Wenn dagegen irgend eine der Functionen $\Psi(\omega) \sin ^{2 \mu-1} \omega$ und ihrer $\mu-1$ ersten Derivirten nach z für einen oder mehrere Werthe (c) der Variablen $\omega$ innerhalb der Integrationsgrenzen unstetig, aber nicht unendlich, wird, so kann man auf das Integral in $(\boldsymbol{A}$.), indem man es durch Einschaltung von Zwischengrenzen an den unstetigen Stellen in Theilintegrale zerlegt, noch immer das Verfahren der theilweisen Integration anwenden, aber es enthält alsdann $S_{m}$ ausser einer Anzahl von Integralen, deren Summe sich für $m=\infty$ wiederum auf $X(+0)$ reducirt, noch eine Anzahl von Gliedern, die vom In-

*) In Betreff der irregulären Functionen, für welche die letzteren Bedingungen nicht erfüllt sind, verweise ich auf eine Abhandlung des Herrn Lipschitz, in Bd. 63 dieses Journals (S. 296-308). 
tegralzeichen frei sind, und weil sie je einen der Werthe von

$$
\frac{\sin \left(m+\frac{1}{2}\right) \omega}{\sin \frac{1}{2} \omega}, \frac{d}{d z}\left(\frac{\sin \left(m+\frac{1}{2}\right) \omega}{\sin \frac{1}{2} \omega}\right), \ldots \frac{d^{\mu-1}}{d z^{\mu-1}}\left(\frac{\sin ^{\prime}\left(m+\frac{1}{2}\right) \omega}{\sin \frac{1}{2} \omega}\right) \quad(\text { für } \omega=c)
$$

als Factor enthalten, bei unbegrenzt wachsendem $m$ unbestimmt oder unendlich werden. Es kann also hier von einer Convergenz der Reihe nicht die Rede sein, und eben so wenig wird dieselbe in dem Falle, wo die Derivirten von $\Psi(\omega)$ stellenweise unendlich grosse Werthe annehmen, stattfinden können, wenngleich sich dieser Fall nicht durch die nämlichen Schlüsse erledigen lässt.

Man darf übrigens nicht übersehen, dass wiewohl nach (6.) die $a$ stetige Functionen der $b$, d. h. (wenn wir $l=1$ setzen) die Grössen $\cos \lambda_{1}$, $\sin \lambda_{1} \cos \lambda_{2}$ etc. stetige Funclionen von $\cos \omega, \sin \omega \cos \omega_{1}$ etc. sind, dennoch für die Variablen $\lambda$ selbst nicht durchweg das Gleiche stattfindet, indem insbesondere $\lambda_{2 \mu+1}$ bei continuirlich sich änderndem $\omega$ plötzlich von einem der Grenzwerthe 0 und $2 \pi$ auf den andern überspringen kann, und diesem Umstande ist es, wie ich beiläufig anführe, zuzuschreiben, dass für das specielle Werthsystem $\varphi_{1}=\varphi_{2}=\cdots=\varphi_{2 \mu+1}=\frac{1}{2} \pi$ die Function $\Psi(\omega)$ bei $\omega=\frac{1}{2} \pi$ im Allgemeinen eine plötzliche Aenderung erfährt und dadurch eine Divergenz der Reihe hervorbringt. Auf der anderen Seite aber ist das Folgende festzuhalten: Wenn die Function $f\left(\lambda_{1}, \ldots \lambda_{2 \mu+1}\right)$ als eine stetige Function $g$ der $2 \mu+2$ Variablen $a_{1}=\cos \lambda_{1}, a_{2}=\sin \lambda_{1} \cos \lambda_{2}$ etc. betrachtet werden kann, und wenn auch die sämmtlichen nach den $a$ genommenen partiellen Differentialquotienten von $g$ bis $\mathrm{zu}$ denen der $\mu-1^{\text {sten }}$ Ordnung inclusive stetige Functionen der $a$ sind, so ist die Function $f$, so wie ihre $\mu-1$ ersten Derivirten nach $\omega$, auch in Bezug auf die Variablen $\omega, \omega_{1}, \ldots$ durchweg stetig, woraus man leicht schliesst, dass dann die Bedingungen erfüllt sind, unter welchen die Transformation von $(A$.) in $(B$.$) möglich ist.$

Was die Reihe $\left(\mathbf{I}^{b}\right.$.) betrifft, so kann man dieselbe offenbar statt bei $m=0$ auch bei $m=-\mu$ beginnen lassen, und wenn man dann jedes Glied derselben durch die Substitution (6.) transformirt, so wird man, unter der Voraussetzung, dass eine $\mu$-malige theilweise Integration stathaft ist, auf ein Integral von der Form

$$
S_{m}=\int_{0}^{\pi} F(\omega)\left(\frac{1}{2} P_{0}(z)+\frac{3}{2} P_{1}(z)+\cdots+\frac{1}{2}(2 m+1) P_{m}(z)\right) \sin \omega d \omega
$$

geführt, dessen Werth, falls $F(\omega)$ endlich, sich nach Dirichlet für $m=\infty$ auf $\boldsymbol{F}(+0)$ reducirt, d. h., wie man zufolge der Bedeutung von $\boldsymbol{F}(\omega)$ finden wird, im Allgemeinen auf $f\left(\varphi_{1}, \ldots \varphi_{2 \mu+2}\right)$.

Journal für Mathematik Bd. LXVI. Heft 2. . 
170 Mehler, Reihenentwicklungen nach Laplaceschen Functionen höherer Ordnung.

\section{S. 4.}

Die Reihen beliebiger Ordnung für Functionen einer einzigen Variablen.

Wenn die Function $f$ nur von der ersten Variablen $\varphi_{1}$ abhängt, während $\mu$ beliebig bleibt, so darf man $\varphi_{2}=\varphi_{3}=\cdots=0$ setzen, und die Reihe $\left(I^{a}\right.$.) lässt sich durch Benutzung einer von Herrn Heine (a. a. O. S. 141) gegebenen Integralformel oder auch durch Benutzung der Differentialgleichung (5.) in die Form bringen:

$$
\begin{aligned}
& S=\frac{2}{\pi} \sum_{\mu}^{\infty} \frac{\Gamma(m-\mu+1)}{m \Gamma^{\prime}(m+\mu)} \frac{d^{\mu} \cos (m \varphi)}{(d \cos \varphi)^{\mu}} \cdot A_{m}, \\
& A_{m}=\int_{0}^{\pi} d \lambda \sin ^{2 \mu} \lambda f(\lambda) \frac{d^{\mu} \cos (m \lambda)}{(d \cos \lambda)^{\mu}},
\end{aligned}
$$

wobei $\varphi, \lambda$ statt $\varphi_{1}, \lambda_{1}$ und $m$ statt des früheren $m+\mu$ geschrieben worden ist. Die Function $\Psi(\omega)$ vereinfacht sich jetzt in

$$
\Psi(\omega)=c \int_{0}^{\pi} f(\lambda) \sin ^{2 \mu-1} \omega_{1} d \omega_{1},
$$

wo $c$ eine leicht angebbare Constante, und nach (6.) ist:

$$
a=\cos \lambda=\cos \varphi \cos \omega-\sin \varphi \sin \omega \cos \omega_{1} .
$$

Ist nun zunächst $\varphi=0$, resp. $\varphi=\pi$, so wird $\lambda=\omega$, resp. $=\pi-\omega$, also von $\omega_{1}$ unabhängig, so dass $\Psi(\omega)$ sich nur durch einen constanten Factor von $f(\lambda)$, resp. $f(\pi-\lambda)$, unterscheidet. Die Reihe ist also für die Grenzwerthe 0 und $\pi$ divergent, wenn die Functionen $f(\lambda), f^{\prime}(\lambda), \ldots f^{(\mu-1)}(\lambda)$ innerhalb des Intervalles von 0 bis $\pi$ nicht sämmtlich stetig sind.

Wenn dagegen $\varphi$ von 0 und $\pi$ verschieden ist, so ist es für alle zwischen 0 und $\pi$ gelegenen Werthe von $\omega$ erlaubt, die Integrationsvariable $\omega_{1}$ durch $a$ auszudrücken, und es wird, wenn man zur Abkürzung setzt:

$$
\begin{gathered}
\sin ^{1-2 \mu} \varphi\left[\sin ^{2} \varphi \sin ^{2} \omega-(\cos \varphi \cos \omega-a)^{2}\right]^{\mu-1}=M, \\
\sin ^{2 \mu-1} \omega \Psi(\omega)=c \int_{\cos (\varphi+\omega)}^{\cos (\varphi-\omega)} f(\arccos a) M d a .
\end{gathered}
$$

Die wiederholte Differentiation dieser Gleichung nach $z=\cos \omega$ ergiebt mit Rücksicht darauf, dass $M$ und die $\mu-2$ ersten Derivirten von $M$ für $a=\cos (\varphi-\omega)$ und $a=\cos (\varphi+\omega)$ verschwinden:

$$
\frac{d^{\alpha} \Psi(\omega) \sin ^{2 \mu-1} \omega}{d z^{\alpha}}=c \int_{\cos (\varphi+\omega)}^{\cos (\varphi-\omega)} f(\arccos a) \frac{d^{\alpha} M}{d z^{\alpha}} d a, \quad(\alpha \leqq \mu-1) .
$$

Dieses Integral aber ist selbst dann eine stetige Function des Parameters $\omega$, 
wenn $f(\arccos a)$ oder $f(\lambda)$ stellenweise discontinuirlich ist, und es verschwindet ausserdem, wenn $\omega$ sich dem Werthe 0 oder $\pi$ unbegrenzt nähert. Diese Eigenschaften reichen hin, um die Umformung der Gleichung $(A$.) des vorigen Paragraphen in $(B$.$) zu rechtfertigen, und die Reihe convergirt also, wenn$ $\varphi$ weder $=0$ noch $=\pi$, gegen den Werth von $X(\varepsilon)$ für ein unendlich kleines positives $\varepsilon$, d. h. gegen $\frac{1}{2}[f(\varphi-\varepsilon)+f(\varphi+\varepsilon)]$, wie man findet, wenn man in der vorangehenden Gleichung $\alpha=\mu-1$ nimmt und die entstehende Formel nach $\omega$ differentiirt.

Die Reihe $\left(\mathbf{I}^{b}\right.$.) nimmt für den Fall einer einzigen Variablen die Form an:

$$
\begin{aligned}
& S=\sum_{\mu}^{\infty} \frac{(2 m+1) \Gamma(m-\mu+1)}{2 \Gamma(m+\mu+1)} \frac{d^{\mu} P_{m}(\cos \varphi)}{(d \cos \varphi)^{\mu}} \cdot B_{m}, \\
& B_{m}=\int_{0}^{\pi} d \lambda \sin ^{2 \mu+1} \lambda f(\lambda) \frac{d^{\mu} P_{m}(\cos \lambda)}{(d \cos \lambda)^{\mu}}
\end{aligned}
$$

und erweist sich dann als im Wesentlichen identisch mit der Entwicklung einer Function nach den Zugeordneten von $P_{m}(\cos \varphi)$. (Vergl. Heine, Handb. d. Kugelf. \$. 52.) Wie ich einer gefälligen Mittheilung des Herrn Heine zu entlehnen mir erlaube, kann diese Reihe und zugleich der Beweis ihrer Convergenz für zwischen 0 und $\pi$ gelegene Werthe von $\varphi$ sehr leicht dadurch erhalten werden, dass man die Kugelfunctionenreihe, durch welche nach Dirichlet jede endliche Function zweier Variablen dargestellt werden kann, auf die specielle Function $F(\varphi, \psi)=f(\varphi) \sin ^{\mu} \varphi \cos (\mu \psi)$ anwendet und die daraus entspringende Entwicklung von dem Factor $\sin ^{\mu} \varphi \cos (\mu \psi)$ befreit. Aber für $\varphi=0$ und $\varphi=\pi$ ist die Division durch diesen Factor unstatthaft, und die Convergenz der Reihe bleibt zweifelhaft. Sie findet jedoch dann allerdings sicher statt, wenn die $\mu-1$ ersten nach $\cos \lambda$ genommenen Differentialquotienten von $f(\lambda) \sin ^{2 \mu} \lambda$, so wie auch diese Function selbst, stetig und an den Grenzen $=0$ sind, und wenn der $\mu^{\text {te }}$ Differentialquotient endlich ist.

\section{S. 5.}

Der Fall einer unendlich grossen Anzahl von Variablen.

Die Summe der unendlichen Reihe auf der rechten Seite von (I.) in $\$$. 2 lässt sich, wenn man dem allgemeinen Gliede den Factor $\varrho^{m}$ hinzufügt und $\varrho<1$ nimmt, durch den folgenden geschlossenen Ausdruck darstellen:

$$
S=\frac{\left(1-\rho^{2}\right) \Gamma_{\frac{1}{2}}(n+1)}{2 \pi^{\frac{1}{2}(n+1)}} \int k^{\prime}\left(1-2 \rho \cos \omega+\rho^{2}\right)^{-\frac{1}{2}(n+1)} d \sigma^{\prime}
$$


172 Mehler, Reihenentwicklungen nach Laplaceschen Functionen höherer Ordnung.

Es bezeichne nun $\nu$ eine ganze Zahl, die kleiner als $n-1$ ist, und es sei $k^{\prime}$ von den Variablen $\lambda_{\nu+1}, \lambda_{\nu+2}, \ldots \lambda_{n}$ unabhängig, dann ist es erlaubt $\varphi_{\nu+1}=$ $\varphi_{\nu+2}=\cdots=0 \mathrm{zu}$ setzen, es wird

$$
\cos \omega=\cos \varphi_{1} \cos \lambda_{1}+\cdots+\sin \varphi_{1} \sin \lambda_{1} \ldots \sin \varphi_{\nu} \sin \lambda_{\nu} \cos \lambda_{\nu+1},
$$

und wenn man die Integrationen nach $\lambda_{\nu+2}, \lambda_{\nu+3}, \ldots$ ausführt, $\cos \lambda_{\nu+1}=t$ und

$$
J=\sqrt{\frac{n}{2 \pi}}\left(1-\varrho^{2}\right) \int_{-1}^{1}\left(1-t^{2}\right)^{-\frac{1}{2}(\nu+3)}\left(\frac{1-2 \varrho \cos \omega+\varrho^{2}}{1-t^{2}}\right)^{-\frac{1}{2}(n+1)} d t
$$

setzt, so ist:

$$
S=\frac{\sqrt{\frac{2}{n}} \Gamma_{\frac{1}{2}}(n+1)}{\pi^{\frac{\lambda}{2}} \Gamma_{\frac{1}{2}}(n-v)} \int_{0}^{\pi} \ldots \int_{0}^{\pi} k^{\prime} J \sin ^{n-1} \lambda_{1} \ldots \sin ^{n-\nu} \lambda_{\nu} d \lambda_{1} \ldots d \lambda_{\nu} .
$$

Wir werden jetzt die bisherigen Variablen vermöge der Substitutionen

$$
\begin{aligned}
\varphi_{1} & =\frac{1}{2} \pi-x_{1} \sqrt{ } \frac{2}{n}, \ldots \varphi_{\nu}=\frac{1}{2} \pi-x_{\nu} / \frac{2}{n}, \\
\lambda_{1} & =\frac{1}{2} \pi-y_{1} / \frac{2}{n}, \ldots \lambda_{\nu}=\frac{1}{2} \pi-y_{\nu} / \frac{2}{n}
\end{aligned}
$$

durch neue ersetzen, ferner statt $k^{\prime}$ die Function $F\left(y_{1}, \ldots y_{\nu}\right)$ einführen, und darauf die Zahl $n$ ins Unendliche wachsen lassen, während $\nu$ einen gegebenen endlichen Werth beibehält, dann geht $S$ über in:

$$
\begin{gathered}
\text { (8.) } \boldsymbol{S}=\frac{1}{\pi^{\frac{1}{2}} \nu} \int_{-\infty}^{\infty} \ldots \int_{-\infty}^{\infty} \boldsymbol{F}\left(y_{1}, \ldots y_{v}\right) J \cdot e^{-Y} d y_{1} \ldots d y_{\nu}, \\
\boldsymbol{Y}=y_{1}^{2}+y_{2}^{2}+\cdots+y_{\nu}^{2},
\end{gathered}
$$

und für $J$ findet man, wenn man $t=\varrho+\vartheta \sqrt{\frac{2}{n}}$ macht, durch eine keiner besonderen Schwierigkeit unterworfene Rechnung den Ausdruck:

worin :

$$
\text { (9.) } \quad J=\left(1-\varrho^{2}\right)^{-\frac{2}{2} v} e^{\frac{2 \rho p-\rho^{2} q}{1-\varrho^{2}}} \text {, }
$$

$$
p=\sum_{s=1}^{s=v} x_{s} y_{s}, \quad q=\sum_{s=1}^{s=v}\left(x_{s}^{2}+y_{s}^{2}\right) .
$$

Es lässt sich nun $J$ auch in die Form bringen:

$$
\left(9^{\prime} .\right) \quad J=\frac{e^{Y} e^{-Q}}{\left(\sqrt{1-\varrho^{2}}\right)^{v}}, \quad \text { wenn: } Q=\sum_{s=1}^{s=v}\left(\frac{\varrho x_{s}-y_{s}}{\sqrt{1-\rho^{2}}}\right)^{2},
$$

und vermöge dieses Werthes von $J$ geht (8.) über in

$$
\text { (10.) } \quad S=\int_{-\infty}^{\infty} \ldots \int_{-\infty}^{\infty} \frac{F\left(y_{1}, \ldots y_{\nu}\right)}{\left(\sqrt{\pi} \sqrt{1-\rho^{2}}\right)^{\nu}} e^{-Q} d y_{1} \ldots d y_{\nu},
$$


und wenn man dieses Integral durch die Substitution

$$
y_{1}=\varrho x_{1}+\sqrt{1-\varrho^{2}} z_{1}, \ldots y_{v}=\varrho x_{\nu}+\sqrt{1-\varrho^{2}} z_{\nu}
$$

transformirt, so findet man, dass dasselbe, wie es auch nach der Art seiner Entstehung nicht anders zu erwarten war, sich für ein unendlich wenig von 1 verschiedenes $\varrho$ der Grenze $F\left(x_{1}, \ldots x_{v}\right)$ nähert, wenigstens sicher dann, wenn die Function $F$ für alle Werthe der Variablen endlich und $F\left(x_{1}, \ldots x_{\nu}\right)$ nicht vieldeutig ist.

Kehren wir jetzt zu (8.) und (9.) zurück und denken uns $J$ in die Reihe $J=J_{0}+J_{1} \varrho+J_{2} \varrho^{2}+\cdots$

entwickelt, welche für $\varrho^{2}<1$ convergirt, so wird unter der Voraussetzung, dass die durch Einführung derselben in (8.) entstehende Entwicklung auch noch für $\varrho=1$ convergent ist, die Function $F$ durch die Reihe

$$
\text { (II.) } \quad F\left(x_{1}, \ldots x_{v}\right)=X_{0}+X_{1}+X_{2}+\cdots
$$

dargestellt, deren allgemeines Glied

$$
X_{m}=\frac{1}{\pi^{\frac{1}{2} \nu}} \int_{-\infty}^{\infty} \ldots \int_{-\infty}^{\infty} F\left(y_{1}, \ldots y_{v}\right) J_{m} e^{-\mathrm{Y}} d y_{1} \ldots d y_{v}
$$

eine ganze Function $m^{\text {ten }}$ Grades der Variablen $x_{1}, \ldots x_{\nu}$ ist und der Differentialgleichung genügt, die aus (5.) durch die Annahme $\varphi_{s}=\frac{1}{2} \pi-x_{s} \sqrt{\frac{2}{n}}$, $n=\infty$ hervorgeht:

$$
\text { (11.) } 2 m \boldsymbol{X}_{m}+\sum_{s=1}^{s=v} e^{x_{s}^{2}} \frac{\partial}{\partial x_{s}}\left(e^{-x_{s}^{2}} \frac{\partial X_{m}}{\partial x_{s}}\right)=0 .
$$

Um nun $J_{m}$ in entwickelter Form darzustellen, zerlege man $J$ in das Product:

$$
\boldsymbol{J}=\boldsymbol{E}\left(x_{1}, y_{1}\right) \boldsymbol{E}\left(x_{2}, y_{2}\right) \ldots E\left(x_{\nu}, y_{v}\right),
$$

wobei $E(x, y)$ definirt ist durch die Gleichung:

$$
\text { (13.) } E(x, y)=\frac{e^{\frac{2 \rho x y-\rho^{2}\left(x^{2}+y^{2}\right)}{1-\varrho^{2}}}}{\sqrt{1-\varrho^{2}}}=\frac{e^{y^{2}} e^{-\frac{(\varrho x-y)^{2}}{1-\varrho^{2}}}}{\sqrt{1-\rho^{2}}} \text {. }
$$

Bei der Entwicklung von $E$ kann man sich entweder der Differentialgleichung

$$
\text { (14.) } \quad 2 \varrho \frac{\partial E}{\partial \varrho}-2 x \frac{\partial E}{\partial x}+\frac{\partial^{2} E}{\partial x^{2}}=0
$$

oder, wie ich es vorziehen werde, des folgenden bestimmten Integrales bedienen, worin $i$ die Bedeutung von $\sqrt{ }-1$ hat:

$$
E(x, y)=\frac{e^{x^{2}}}{\sqrt{\pi}} \int_{-\infty}^{\infty} e^{-(t-y i)^{2}-\left(x+\varrho t^{2}\right)^{2}} d t
$$


174 Mehler, Reihenentwicklungen nach Laplaceschen Functionen höherer Ordnung.

Wenn man hierin die Exponentialgrösse mittels des Taylorschen Lehrsatzes nach Potenzen von $\rho$ entwickelt und

$$
\begin{aligned}
& \text { (16.) } x^{(\alpha)}=(-2)^{-\alpha} e^{x^{2}} \frac{d^{\alpha} e^{-x^{2}}}{d x^{\alpha}}, \\
& \text { (17.) } y^{(\alpha)}=\frac{i^{-\alpha}}{\sqrt{ } \pi} \int_{-\infty}^{\infty} e^{-(1-y i)^{2}} t^{\alpha} d t
\end{aligned}
$$

setzt, wobei $y^{(\alpha)}$ genau dieselbe Function von $y$, wie $x^{(\alpha)}$ von $x$, ist, so ergiebt sich:

$$
\text { (18.) } \quad E(x, y)=\sum_{\alpha=0}^{\alpha=\infty} \frac{2^{\alpha} \varrho^{\alpha}}{\Pi(\alpha)} x^{(\alpha)} y^{(\alpha)},
$$

und hieraus folgt wegen (12.):

$$
J_{m}=\Sigma \frac{2^{\alpha_{1}} x_{1}^{\left(\alpha_{1}\right)} y_{1}^{\left(\alpha_{1}\right)}}{\Pi\left(\alpha_{1}\right)} \frac{2^{\alpha_{2}} x_{2}^{\left(\alpha_{2}\right)} y_{2}^{\left(\alpha_{2}\right)}}{\Pi\left(\alpha_{2}\right)} \cdots \frac{2^{\alpha_{\nu}} x_{\nu}^{\left(\alpha_{\nu}\right)} y_{\nu}^{\left(\alpha_{\nu}\right)}}{\Pi\left(\alpha_{\nu}\right)},
$$

wenn die Summe sich auf alle Werthe $0,1,2,3 \ldots$ der $\alpha$ bezieht, für welche $\alpha_{1}+\cdots+\alpha_{\nu}=m$. Vermöge dieses Ausdruckes verwandelt sich die Reihe (II.) in

$$
\text { (III.) } \begin{aligned}
\quad \boldsymbol{F}\left(\boldsymbol{x}_{1}, \ldots \boldsymbol{x}_{\nu}\right) & =\sum \frac{2^{\alpha_{1}+\ldots+\alpha_{\nu}} \cdot \boldsymbol{A}_{\alpha_{1}, \ldots \alpha_{\nu}}}{\pi^{\frac{1}{2} \nu} \boldsymbol{\Pi}\left(\boldsymbol{\alpha}_{1}\right) \ldots \boldsymbol{\Pi}\left(\boldsymbol{\alpha}_{\nu}\right)} x_{1}^{\left(\alpha_{1}\right)} \ldots x_{\nu}^{\left(\alpha_{\nu}\right)}, \\
A_{\alpha_{1}}, \ldots \alpha_{\nu} & =\int_{-\infty}^{\infty} \ldots \int_{-\infty}^{\infty} F\left(y_{1}, \ldots y_{\nu}\right) y_{1}^{\left(\alpha_{1}\right)} \ldots y_{\nu}^{\left(\alpha_{\nu}\right)} e^{-\gamma} d y_{1} \ldots d y_{\nu},
\end{aligned}
$$

während für $\alpha_{1}, \ldots \alpha_{\nu}$ alle Zahlen der Reihe $0,1,2,3, \ldots$ zu setzen sind.

In dieser Form erweist sich unsere Entwicklung leicht als ein specieller Fall der von Herrn Hermite gegebenen, auf welche in der Einleitung hingedeutet wurde. Um auch $\mathrm{zu}$ diesen allgemeineren Reihen in ähnlicher Weise zu gelangen, wie wir zu (III.) gelangt sind, müsste man auf (8.) und (10.) zurückgehen und in $Q$ statt der Summe der Quadrate der Grössen $\rho x_{1}-y_{1}, \rho x_{2}-y_{2}, \ldots$ eine beliebige positive quadratische Form eben derselben Grössen einführen. Man wird dadurch zunächst auf eine einzige, der Reihe (II.) analoge Reihe geführt. Stellt man darauf das verallgemeinerte $J$ in $\left(9^{\prime}.\right)$, um es nach Potenzen von $\varrho$ entwickeln zu können, durch ein $\nu$-faches Integral dar, in ähnlicher Weise, wie in unserem speciellen Falle $\boldsymbol{J}$ vermöge (12.) und (15.) durch ein Product von $\nu$ einfachen Integralen dargestellt wurde, so erhält man für $J_{m}$ eine Zerfällung in einfachere Bestandtheile, deren jeder das Product einer Function der $x$ in eine davon verschiedene Function der $y$ ist. Da aber der Entwicklungscoefficient $J_{m}$ in Wahrheit in Bezug auf 
die $x$ und $y$ symmetrisch ist, so ergiebt sich durch die Vertauschung dieser Grössen noch eine zweite, von der ersten verschiedene Darstellungsform desselben, und auf diese Weise entstehen die beiden von Herrn Hermite gegebenen Entwicklungen, deren inniger Zusammenhang auch hierdurch aufs Deutlichste hervortritt.

Zum Schlusse mögen noch einige Notizen über die Function $x^{(\alpha)}$ Platz finden. Durch das Integral in (17.) erhält man sehr leicht die bekannten Relationen:

$$
\frac{d x^{(\alpha)}}{d x}=\alpha x^{(\alpha-1)}, \quad x x^{(\alpha)}=x^{(\alpha+1)}+\frac{\alpha}{2} x^{(\alpha-1)} .
$$

Die zweite ist ein specieller Fall der folgenden:

$$
x^{(\alpha)} x^{(\beta)}=x^{(\alpha+\beta)}+\frac{\alpha \beta}{2} x^{(\alpha+\beta-2)}+\frac{\alpha(\alpha-1) \beta(\beta-1)}{2.4} x^{(\alpha+\beta-4)}+\cdots
$$

Nach Potenzen von $x$ geordnet ist:

$$
x^{(\alpha)}=x^{\alpha}-\frac{\alpha(\alpha-1)}{1} \frac{x^{\alpha-2}}{2^{2}}+\frac{\alpha(\alpha-1)(\alpha-2)(\alpha-3)}{1.2} \frac{x^{\alpha-4}}{2^{4}}-\cdots
$$

Den Werth des Polynoms $x^{(\alpha)}$ für ein unendlich grosses $\alpha$ hat Herr Hermite mit Hülfe der Differentialgleichung

$$
\frac{d^{2} x^{(\alpha)}}{d x^{2}}-2 x \frac{d x^{(\alpha)}}{d x}+2 \alpha x^{(\alpha)}=0
$$

bestimmt. Um denselben vermittelst des bestimmten Integrales in (17.) abzuleiten, bringe man dasselbe in die Form:

$$
\left(17^{\prime} .\right) \quad x^{(\alpha)}=\frac{2}{\sqrt{ } \pi} e^{x^{2}} \int_{0}^{\infty} e^{-t^{2}} t^{\alpha} \cos \left(2 t x-\frac{1}{2} \alpha \pi\right) d t
$$

und transformire es durch die Substitution $t=\sqrt{\frac{\alpha}{2}}+\vartheta$, so wird:

$$
\begin{gathered}
x^{(\alpha)}=K \int_{-\sqrt{2} \alpha}^{\infty} e^{-\vartheta^{2}-\theta} \cos \left(2 x \vartheta+x \sqrt{2 \alpha}-\frac{1}{2} \alpha \pi\right) d \vartheta \\
K=\frac{2}{\sqrt{\pi}} e^{-\frac{1}{2} \alpha}\left(\frac{\alpha}{2}\right)^{\frac{1}{2} \alpha} e^{x^{2}}, \quad \theta=\vartheta \sqrt{2 \alpha}-\alpha \log \left(1+\vartheta \sqrt{\frac{2}{\alpha}}\right) .
\end{gathered}
$$

Das in $K$ multiplicirte Integral zerlege man in drei andere $T_{1}, T_{2}, T_{3}$ mit den Grenzen $-\sqrt{\frac{\alpha}{2}}$ und $-m,-m$ und $m, m$ und $\infty$, wobei $m$ positiv und $<\sqrt{\frac{\alpha}{2}}$. Da $\theta$ innerhalb der Integrationsgrenzen beständig positiv ist, so sind 
176 Mehler, Reihenentwicklungen nach Laplaceschen Functionen höherer Ordnung.

$T_{1}$ und $T_{3}$ verschwindend klein, sofern nur $m$ mit $\alpha$ zugleich unendlich wird. Man kann aber $m$ so langsam zunehmen lassen, dass in $T_{2}$ beständig $\theta=\vartheta^{2}$ ist; es wird also:

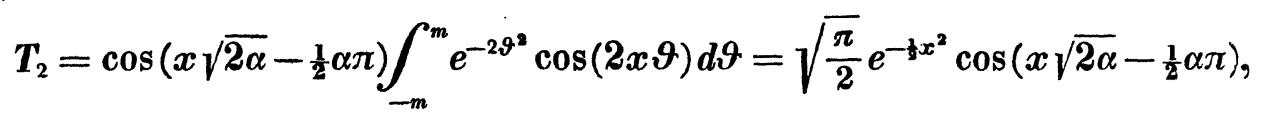

und somit:

$$
x^{(\alpha)}=\sqrt{2} e^{-\frac{1}{2} \alpha}\left(\frac{\alpha}{2}\right)^{\frac{1}{2} \alpha} e^{\frac{1}{2} x^{2}} \cos \left(x \sqrt{2 \alpha}-\frac{1}{2} \alpha \pi\right) .
$$

Danzig, im Mai 1866. 